\title{
Modeling power flow in the induction cavity with a two dimensional circuit simulation
}

\author{
Fan Guo," Wenkang Zou, Boyi Gong, Jihao Jiang, Lin Chen, Meng Wang, and Weiping Xie \\ Key Laboratory of Pulsed Power, Institute of Fluid Physics, China Academy of Engineering Physics, \\ P.O. Box 919-108, Mianyang 621900, China
}

(Received 28 May 2016; published 21 February 2017)

\begin{abstract}
We have proposed a two dimensional (2D) circuit model of induction cavity. The oil elbow and azimuthal transmission line are modeled with one dimensional transmission line elements, while 2D transmission line elements are employed to represent the regions inward the azimuthal transmission line. The voltage waveforms obtained by $2 \mathrm{D}$ circuit simulation and transient electromagnetic simulation are compared, which shows satisfactory agreement. The influence of impedance mismatch on the power flow condition in the induction cavity is investigated with this $2 \mathrm{D}$ circuit model. The simulation results indicate that the peak value of load voltage approaches the maximum if the azimuthal transmission line roughly matches the pulse forming section. The amplitude of output transmission line voltage is strongly influenced by its impedance, but the peak value of load voltage is insensitive to the actual output transmission line impedance. When the load impedance raises, the voltage across the dummy load increases, and the pulse duration at the oil elbow inlet and insulator stack regions also slightly increase.
\end{abstract}

DOI: 10.1103/PhysRevAccelBeams.20.020401

\section{INTRODUCTION}

An induction voltage adder (IVA) is widely used for many applications including flash radiography [1,2], detection of fissile material [3,4] and gamma-ray simulation [5]. Presently, many IVAs are being operated or built around the world such as Hermes III [6], Saber [7], Mercury [8], Radiographic Integrated Test Stand (RITS) [9-11], Cygnus [12] in U.S., Hydrus [13] in Atomic Weapons Establishment (AWE) in U.K. and Jianguang accelerator [14] in Northeast Institute of Nuclear Technology (NINT) in China for various research aims. The most powerful IVA machine to date is Hermes III which could deliver $20 \mathrm{MV}, 750 \mathrm{kA}, 40$ ns pulse to the diode [15].

Usually, the IVA machine is composed of a Marx generator, pulse forming section and induction cavity [16]. The stored energy in the Marx generator is discharged relatively slowly into the pulse forming section. The voltage pulse with fast rise time could be achieved since the switch in the pulse forming section is designed with low inductance, and a laser triggered gas switch is frequently employed to reduce the mistiming of pulse forming sections. The pulse forming section operates at a relatively low voltage. Therefore, the insulation volume and cost of each section are reduced, and it can also advance reliability.

\footnotetext{
*Corresponding author. 394560789@qq.com

Published by the American Physical Society under the terms of the Creative Commons Attribution 3.0 License. Further distribution of this work must maintain attribution to the author $(s)$ and the published article's title, journal citation, and DOI.
}

A much higher voltage output pulse could be achieved by connecting the pulse forming sections in series with identical induction cavities. Its bore is threaded by a central conductor that together with the cylindrical surface of the bore forms an output transmission line. The summed high voltage is restricted to the output transmission line $[17,18]$.

It is an ideal design that the electromagnetic wave could transmit from the Marx generator into the load without any reflection [19]. However, because of insulation restriction and complex structure transformation impedance mismatches in the induction cavity are inevitable. The impedance mismatches and resultant reflections not only reduce the energy and power efficiencies of the machine, but also increase the operating voltage in the induction cavity. These make the cavity designing complicated and challenging. For instance, the maximum output voltage of each induction cavity in the Mercury machine is about $1.0 \mathrm{MV}$, but the insulator stack and radial feed gap regions must handle a 1.3 MV spike [20]. This voltage overshoot determines the length of insulator stack and the shape of cathode plate, which should be accurately evaluated to make sure insulation is reliable. Therefore, it is necessary to investigate the influence of impedance mismatch on the power flow condition in the induction cavity.

Circuit simulation tools are usually used to estimate the operating parameters such as peak voltage, pulse duration and amplitude of conduction current for each component in the induction cavity $[21,22]$. They provide the designing criteria for each component. However, as the azimuthal transmission line is frequently employed in the induction cell in order to make the magnetic field uniform at the output transmission line section, there exist two orthogonal 
directions for the power flow when the electrical pulse flows inward the azimuthal transmission line. The power flow in the cavity transmits in the radial and azimuthal directions at the insulator stack and radial feed gap sections, and at the output transmission line section it transmits in the axial and azimuthal directions. Meanwhile, the output voltage rise time $(10 \%-90 \%)$ of the pulse forming section is usually comparable to the azimuthal transit time of the induction cell. A two dimensional (2D) circuit model should be proposed to take this $2 \mathrm{D}$ power flow into account [23-25].

In this paper, we describe a $2 \mathrm{D}$ circuit model of the induction cavity. 2D transmission line elements are used to model the power flow inward the azimuthal transmission line. The influence of impedance mismatch among the azimuthal transmission line, output transmission line and the load on the operating voltages in the induction cavity will be investigated with the circuit model. This article is organized as follows. The 2D circuit model will be given in detail in the next section. A full size transient electromagnetic model is proposed to verify the exactness of this circuit model. The results obtained by circuit simulation and transient electromagnetic simulation will be compared and given in Sec. III. In Sec. IV, the influence of impedance mismatch on the power flow condition in the induction cavity will be shown. A short summary and discussion are presented in Sec. V.

\section{2D CIRCUIT MODEL OF THE INDUCTION CAVITY}

\section{A. General configuration of the induction cavity}

Figure 1 shows the cross section view of a single-point feed induction cavity which is operated in positive polarity. It could be easily converted to negative polarity by inserting the center conductor from the other side or by rotating the

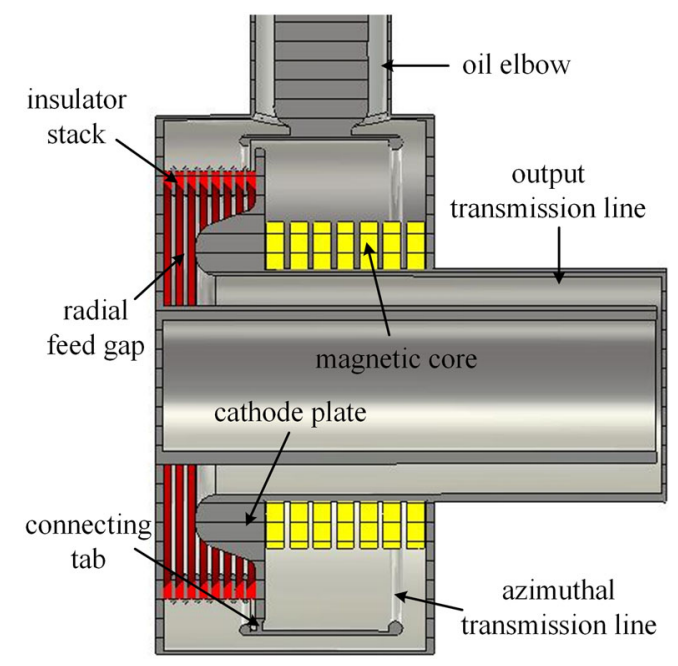

FIG. 1. Schematic cross section of an induction cavity which mainly includes oil elbow, azimuthal transmission line, connecting tab, insulator stack, radial feed gap, cathode plate, magnetic core and output transmission line. induction cavity 180 degrees. The electrical pulse generated by the pulse forming section transmits across the oil elbow into the induction cavity and then flows in opposite directions through the azimuthal transmission line. The cathode plate is electrically connected with the azimuthal transmission line at certain parts of the entire circumference with tabs. The configuration of the connecting tab and impedance profile of the azimuthal transmission line are often carefully optimized to make the magnetic field uniform at the output transmission line section, as the magnetic field distribution nonuniform will perturb the electron flow in the magnetically insulated transmission line (MITL) and increase the induction cell equivalent inductance [26,27]. The magnetic core is used to reduce the current flowing from the high voltage electrode to the ground which is termed as loss current. This material usually takes up the largest region in the induction cell. The insulator stack serves as a barrier between the oil and vacuum regions. Because the insulator stack must operate at the relatively low electric field and have a large highvoltage-to-ground spacing, the radial feed gap is designed to reduce the cell inductance and help grade the voltage uniformly along the insulator stack. This section is also able to shield the insulator stack from electrons traveling radially outward if the output transmission line operates as MITL and debris from the diode moving to the pulsed power machine. The high voltage pulses are added to the output transmission line [28].

\section{B. Description of the 2D circuit model}

The 2D circuit model of the induction cell is created in the cylindrical system and is shown in Fig. 2. The $R, \theta$ and $Z$ directions are defined as radial, azimuthal and axial directions, respectively. The power flow at the oil elbow section transmits only in the radial direction. Thus, the one dimensional (1D) transmission line element is needed to represent the propagation process of the electromagnetic wave. At the azimuthal transmission line part, the power flow transmits in the azimuthal direction. The mesh cell size in the $\theta$ direction is uniform and 10 degrees around the circumference. Therefore, the azimuthal transmission line is characterized by $361 \mathrm{D}$ transmission line elements with the same impedance and transit time. Because the width of the azimuthal transmission line high voltage electrode is shorter than the length of the induction cell, the azimuthal transmission line impedance is estimated as [29]

$$
Z=\frac{377}{\sqrt{\varepsilon_{r}}} \frac{1}{w / d+1.393+0.667 \ln (w / d+1.444)},
$$

where $\varepsilon_{r}$ is the relative permittivity of insulation medium which is always the transformer oil, $w$ is the width of the azimuthal transmission line high voltage electrode, and $d$ is the radial distance between the high voltage electrode and the inner cylindrical surface of induction cell. 


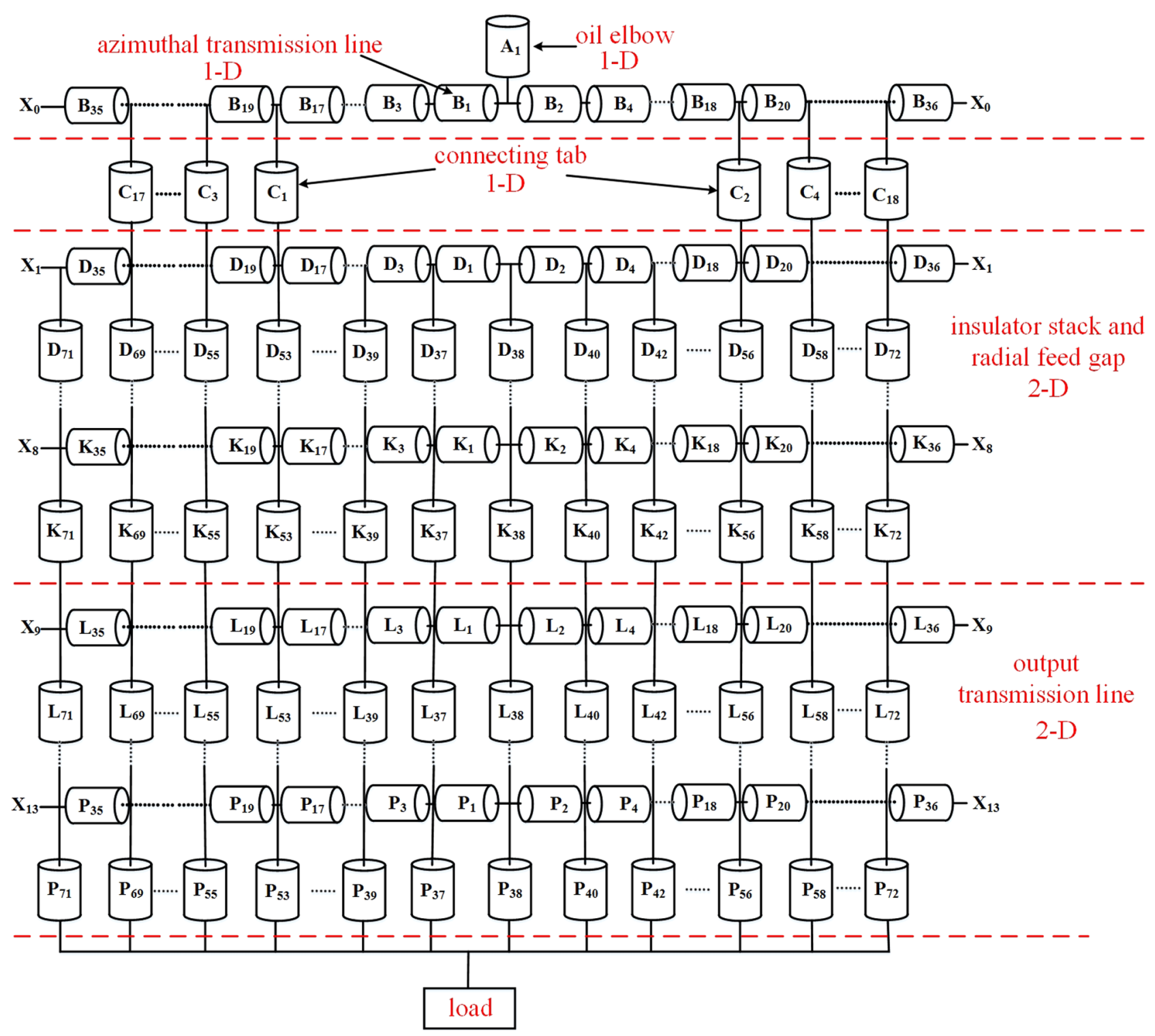

FIG. 2. Block diagram showing the 2D circuit model topology of induction cavity. The oil elbow, azimuthal transmission line, and connecting tab are modeled with 1D transmission line elements. 2D circuit elements are used to represent the regions inward the azimuthal transmission line. The transmission line element labeled $A_{1}$ is used to model the oil elbow, and the elements named $B_{1}$ to $B_{36}$ indicate the azimuthal transmission line. The connecting tab is modeled with transmission line elements labeled $C_{1}$ to $C_{18}$. The insulator stack and radial feed gap regions are modeled with transmission line elements labeled $D$ to $K$ with subscript 1 to 72 . The elements with subscript 1 to 36 are the azimuthal elements, and the elements with subscript 37 to 72 represent the radial elements. The transmission line elements labeled $L$ to $P$ with subscript 1 to 72 denote the output transmission line section. The elements with subscript 1 to 36 are the azimuthal elements, and the elements with subscript 37 to 72 represent the axial elements. The junctions named $X_{0}$ to $X_{13}$ indicate that the two junctions with the same letter are electrically connected together in the circuit model. It should be noted that this circuit model is associated with the type of connecting tab displayed in Fig. 3(a). The transit time of output transmission line is assumed to be $5.0 \mathrm{~ns}$, and it could be easily adjusted according to the actual length of output transmission line.

The transit time $\tau$ along the whole azimuthal transmission line is calculated according to

$$
\tau=\frac{L_{a}+L_{c}}{2 c} \sqrt{\varepsilon_{r}}
$$

where $L_{a}$ is the perimeter of the inner cylindrical surface of induction cavity, $L_{c}$ is the perimeter of the azimuthal transmission line high voltage electrode. This assumes that the electromagnetic wave propagates along a path which is the average of $L_{a}$ and $L_{c}$ [30]. $c$ represents the speed of light in vacuum.

Since the width of connecting tab in the radial direction is very short, the power flow transmitting in the azimuthal direction could be neglected at this section. It is modeled 


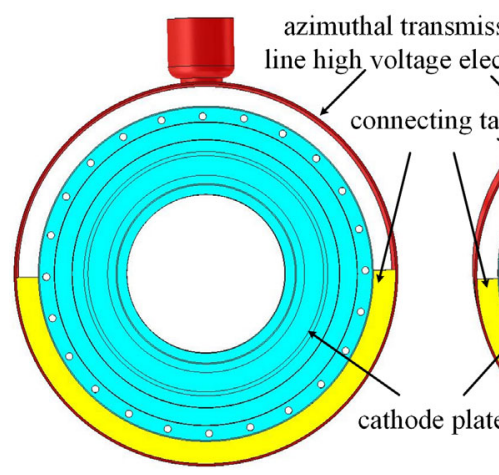

(a)

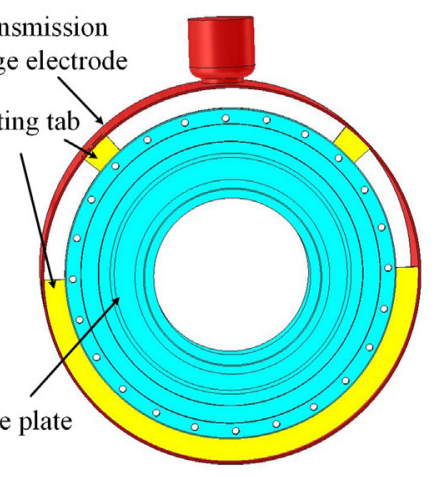

(b)
FIG. 3. Two examples of connecting tab configuration. (a) One continuous tab on the lower half circle allows conduction from the azimuthal transmission line to cathode plate, (b) there are two tabs on the upper half circle extending from \pm 40 degrees to \pm 50 degrees and one continuous tab on the lower half circle. The angles are measured in each direction from the midpoint of the oil elbow. The connecting tab forces the current injected onto the azimuthal transmission line to spread away from the feed port.

with the 1D transmission line element which could only represent the radial directional power flow. The mesh cell size is the same as that of the azimuthal transmission line. Two typical kinds of connecting tab are displayed in Fig. 3, which have already been tested and employed in the RITS machine [31]. The configuration of the connecting tab displayed in Fig. 3(a) is characterized by 18 1D transmission line elements, and two more elements must be employed to model the connecting tab displayed in Fig. 3(b). These two elements are electrically connected with the front ends of corresponding azimuthal transmission line elements labeled $B_{9}$ and $B_{10}$ and the corresponding insulator stack transmission line elements labeled $D_{9}$ and $D_{10}$.

The transmission line element impedance is approximately evaluated from the formula for the transmission line in parallel plate geometry which is accurate for small regions,

$$
Z=\frac{377}{\sqrt{\varepsilon_{r}}} \frac{H}{D},
$$

where $H$ is the high-voltage-to-ground spacing in the axial direction, and $D$ is the mean width of electrodes in the $R$ direction for each mesh cell.

At the insulator stack and radial feed gap regions, the mesh cell size in the $\theta$ direction is also uniform and 10 degrees around the circumference, while eight nonuniform cells are employed in the $R$ direction. Each mesh cell possesses one radial element and one azimuthal element which model the power flow in the $R$ and $\theta$ directions, respectively. The radial element impedance is calculated by

$$
Z=36 \times \frac{60}{\sqrt{\varepsilon_{r}}} \frac{H}{r} .
$$

The quantity $r$ is the mean radius of electrodes in the $R$ direction for each mesh cell. The leading factor of 36 in Eq. (4) is related to modeling the radial transmission line with 36 parallel elements. The azimuthal element is also treated as a parallel plate transmission line. There are 288 radial elements and 288 azimuthal elements in total to model the insulator stack and radial feed gap regions.

At the output transmission line region, the mesh cell sizes in the $\theta$ and $Z$ directions are 10 degrees and $33.3 \mathrm{~cm}$, respectively. The axial element impedance is calculated by the following formula:

$$
Z=36 \times 60 \ln \frac{R_{a}}{R_{b}}
$$

where $R_{a}$ and $R_{b}$ are the radius of anode and cathode of the output transmission line, respectively. The leading factor of 36 in Eq. (5) is also related to modeling the coaxial output transmission line by 36 parallel elements. The azimuthal element impedance is evaluated according to Eq. (3) too.

\section{VALIDATION WITH TRANSIENT ELECTROMAGNETIC SIMULATION}

A full size transient electromagnetic simulation is employed to check out the accuracy of this 2D circuit model. The voltage pulses acquired by transient simulation and circuit simulation are compared.

\section{A. Description of the transient electromagnetic model}

During the transient electromagnetic model, the width of the azimuthal transmission line high voltage electrode is set to be $45 \mathrm{~cm}$ and the radial distance between the high voltage electrode and the ground is $5.0 \mathrm{~cm}$. The relative permittivity of transformer oil is assumed to be 2.3 . Therefore, the corresponding impedance of azimuthal transmission line is about $20.79 \Omega$ according to Eq. (1). The transit time of the half circle azimuthal transmission line is $12.4 \mathrm{~ns}$. The relative permittivity of the insulator stack is about 2.54 for Rexolite which is commonly used as an insulation material in a pulsed power machine. The output transmission line impedance is set to be $20.0 \Omega$ and the transit time from the ground end to the load region is $5.0 \mathrm{~ns}$. The induction cell is terminated by a $7.0 \Omega$ dummy load.

The dynamic characteristics of magnetic core could not be modeled in the transient electromagnetic simulation, and this component is modeled ideally here simply placing material with relative permeability of 1000 . Thus the energy loss in the magnetic core could not be taken into consideration. However, this modeling method has been proven to be an effective way and could reproduce the experimental results [14,32]. Actually, the shunting resistance of the magnetic core is time dependent and could be evaluated by the following expression [33]: 


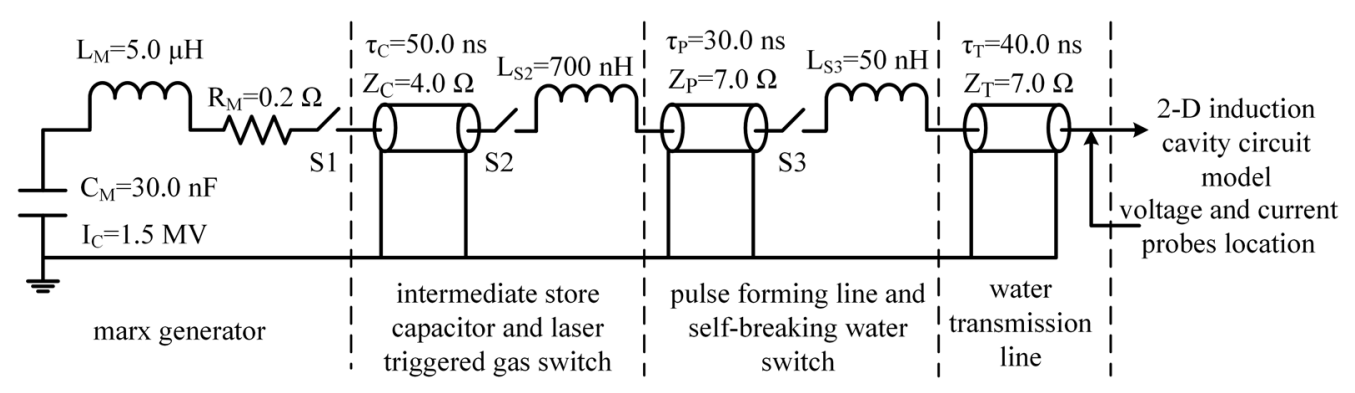

FIG. 4. A pulse generation circuit is used to drive the $2 \mathrm{D}$ circuit model of induction cavity which includes Marx generator, intermediate store capacitor, laser triggered gas switch, PFL, self-breaking water switch and water transmission line. The laser triggered gas switch and self-breaking water switch are modeled with ideal elements without consideration of capacitance and resistance. The oil prepulse switch is also not taken into account. The quantities $I_{C}, C_{M}, L_{M}$ and $R_{M}$ are the equivalent charging voltage, capacitance, inductance and resistance of the Marx generator, respectively. $S 1, S 2$ and $S 3$ represent the switches in the Marx generator, laser triggered gas switch and self-breaking water switch, respectively. $\tau_{C}$ and $Z_{C}$ are the transit time and impedance of the intermediate store capacitor, respectively. $L_{S 2}$ is the inductance of laser triggered gas switch. $\tau_{P}$ and $Z_{P}$ are the transit time and impedance of the PFL, respectively. $L_{S 3}$ is the inductance of self-breaking water switch. $\tau_{T}$ and $Z_{T}$ are the transit time and impedance of the water transmission line, respectively.

$$
R_{\text {cores }}(t)=\frac{2 S\left(\mu \mu_{0} \rho_{\text {cores }}\right)^{1 / 2}}{\pi^{3 / 2} r_{\text {cores }} \delta t^{1 / 2}} .
$$

The quantity $S$ is the total cross-sectional area of the magnetic core within one induction cavity which is $490 \mathrm{~cm}^{2}$ in our simulation model. $\mu$ and $\mu_{0}$ are the relative permeability of magnetic core and permeability of free space, respectively. $\mu$ is dependent on the magnetization rate which is about 10 to $50 \mathrm{~T} / \mu \mathrm{s}$ for the IVA machine. Metglas is usually used as a magnetic core material in the induction cavity, and $\mu$ is in the range of one hundred to several hundred according to previous experimental results [34]. $\rho_{\text {cores }}$ is the electrical resistivity of the magnetic core that is about $123 \mu \Omega \mathrm{cm} . r_{\text {cores }}$ is the radius of the centroid of the magnetic core, and it is set to be $42 \mathrm{~cm}$ in our simulation model. $\delta$ is the thickness of Metglas tape used to fabricate the core which is commonly about $23 \mu \mathrm{m}$. It is seen that the shunting resistance $R_{\text {cores }}(t)$ decreases with the time variable $t$ during the discharge process. As the voltage pulse duration is usually shorter than $100 \mathrm{~ns}$ for most of the IVA machines, the shunting resistance is estimated to be sufficiently greater than the load impedance during this period of time. This also should be the case if the IVA is well designed.

\section{B. Obtaining the forward going voltage pulse for transient electromagnetic simulation}

The feed voltage pulse for transient electromagnetic simulation is acquired by the following method. A pulse generation circuit as shown in Fig. 4 is connected to the 2D circuit model of induction cavity. This pulse generation section has similar components like the RITS machine. A Marx generator charges a coaxial water intermediate store capacitor which is switched out by a laser triggered gas switch to the pulse forming line (PFL). The PFL is discharged by a self-breaking water switch to a matched water transmission line which has 80.0 ns two-way transit time. The voltage pulse generated by PFL transmits through the water transmission line and drives the induction cavity. The voltage $V$ and current $I$ at the inlet of induction cell are observed. The forward going voltage wave $V_{f}$ at this position can be obtained as

$$
V_{f}=\frac{V+I Z}{2} .
$$

The quantity $Z$ is the corresponding characteristic impedance at the probe location. The calculated forward going voltage wave has a peak value about $1.61 \mathrm{MV}$, a rise time about $7.3 \mathrm{~ns}$ and full-width-at-half-maximum (FWHM) about 65 ns. This forward going voltage pulse is injected into the induction cavity during the transient electromagnetic simulation.

\section{Simulation results}

The oil elbow inlet voltage obtained by 2D circuit simulation and transient electromagnetic simulation are compared and shown in Fig. 5. It is seen that the voltage waveforms agree with each other very well not only at the rising edge but also for the tail of the pulse. The PFL output voltage obtained by $2 \mathrm{D}$ circuit simulation is also shown in Fig. 5. The amplitude is much less than the maximum operating voltage at the oil elbow inlet which is about 2.05 MV. As the water transmission line matches the PFL section, the evident voltage increasing at the oil elbow inlet should be resulted from impedance mismatch between the induction cavity and water transmission line. In fact, previous investigation results also indicated that the induction cavity inlet voltage was strongly influenced by the value of azimuthal transmission line impedance. As the azimuthal transmission line impedance increases, the maximum voltage at the induction cavity inlet raises [32]. This should be 


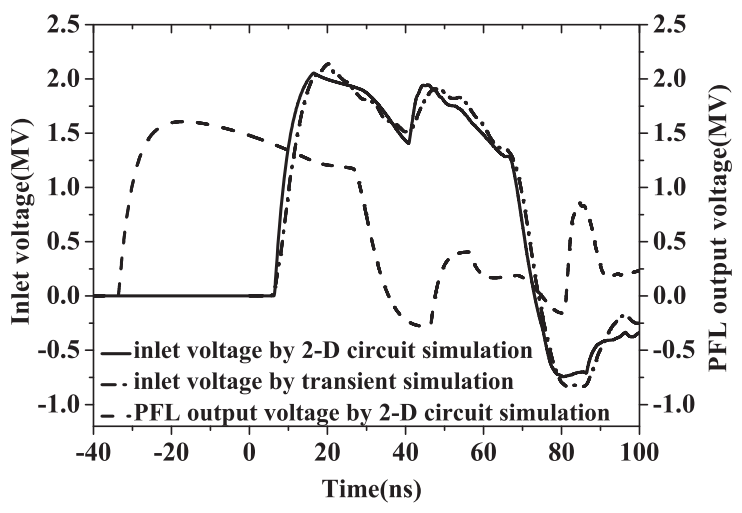

FIG. 5. The oil elbow inlet voltage waveforms acquired by 2D circuit simulation (black solid line) and transient electromagnetic simulation (black dash-dotted line). The PFL output voltage obtained by $2 \mathrm{D}$ circuit simulation is also presented (black dashed line).

taken into account when designing the output barrier separating the water section and the oil section.

The azimuthal transmission line operating voltage at 0 degree, 90 degrees and 180 degrees obtained by 2D circuit simulation and transient electromagnetic simulation is shown in Fig. 6. The voltage waveforms are also able to be in line with each other very well. The rise time of voltage pulse at 0 degree is about $25.1 \mathrm{~ns}$, and decreases to 14.6 and $6.7 \mathrm{~ns}$ at 90 and 180 degrees, respectively. The maximum voltage at this section is about $2.5 \mathrm{MV}$ and even higher than that of oil elbow inlet. This may be due to the fact that the transit time of azimuthal transmission line is able to compare to the rise time of forward going injected voltage pulse. The azimuthal transmission line should not be treated as a lumped inductor. As the power flow propagation along the azimuthal transmission line has two parallel paths extending away from the feed port, the actual characteristic impedance of the azimuthal transmission line in both directions should be halved [28]. This value is smaller than the output transmission line impedance. The total operating voltage is the superposition of forward going and reflected waves with the same polarity.

The insulator stack voltage achieved by the two different methods at 0,90 , and 180 degrees are displayed in Fig. 7. The peak voltage at this section is about $2.4 \mathrm{MV}$. The rise time of voltage pulse at 0,90 , and 180 degrees are 21.6, 14.5, and 9.43, respectively. It is clear that the voltage waveforms are gradually sharpened around the circumference. The insulator stack voltage obtained by 1D circuit simulation is also shown in Fig. 7(a). The corresponding maximum voltage is about $2.2 \mathrm{MV}$ and is a little lower than the results given by $2 \mathrm{D}$ circuit simulation and transient electromagnetic simulation. Because the azimuthal directional power flow inward the azimuthal transmission line is not able to be modeled by $1 \mathrm{D}$ circuit simulation, only one insulator stack voltage waveform could be given.
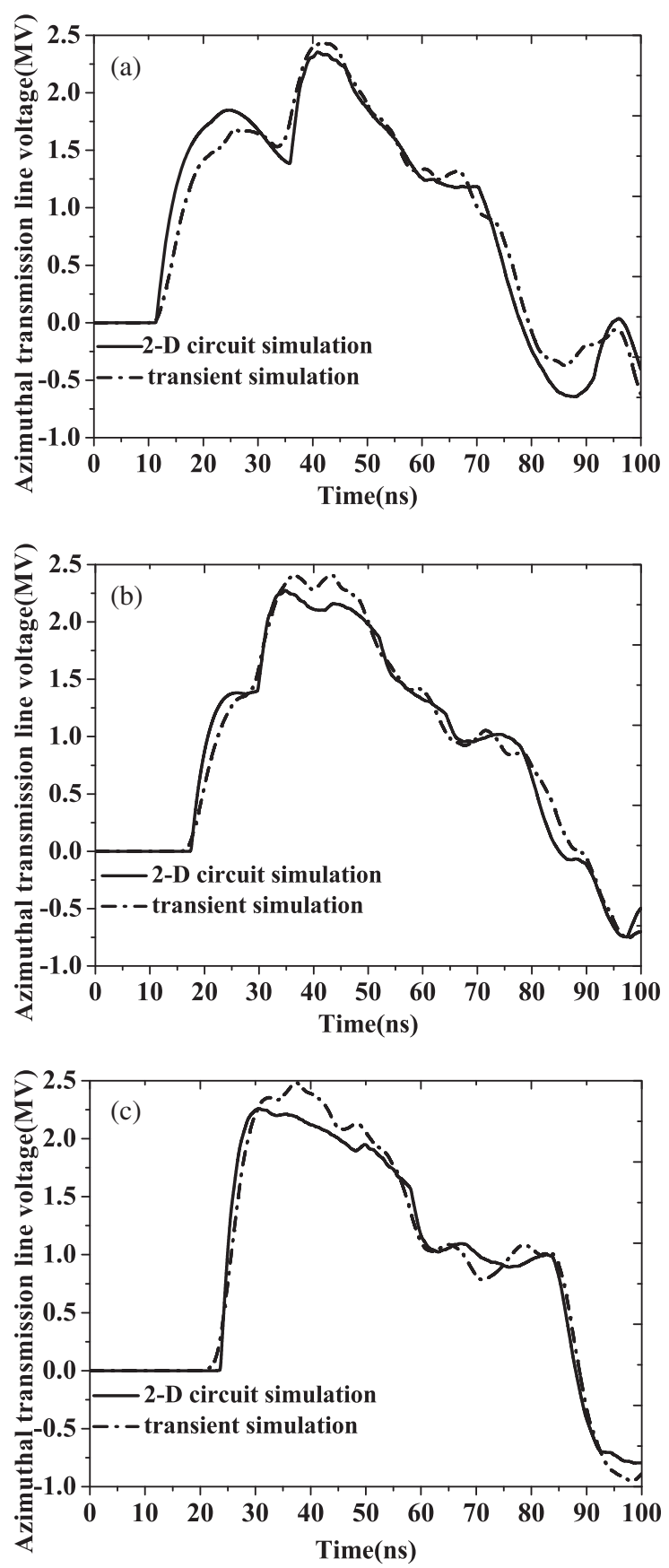

FIG. 6. Azimuthal transmission line voltage waveforms obtained by $2 \mathrm{D}$ circuit simulation (black solid line) and transient electromagnetic simulation (black dash-dotted line) at (a) 0 degree, (b) 90 degrees and (c) 180 degrees.

The insulator stack is close to the radial feed gap region. The voltage waveforms at these two regions should be approximately the same. The peak voltage at the radial feed gap determines the maximum electric field on the cathode plate. This value should not exceed the explosive electron emission threshold. A better design usually keeps the maximum electric field less than $200 \mathrm{kV} / \mathrm{cm}$ on any negative cathode plate surface, and the hard anodize 

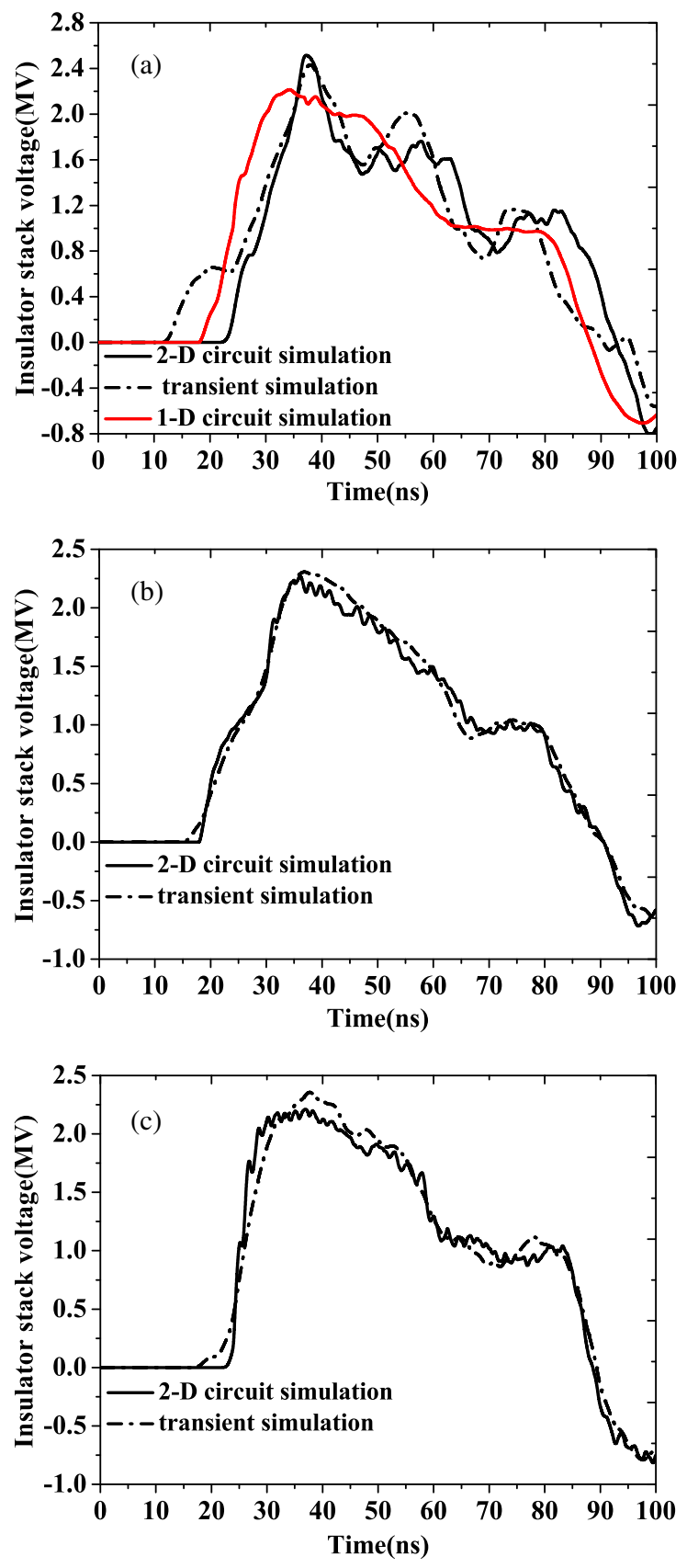

FIG. 7. Insulator stack voltage waveforms obtained by the two different methods at (a) 0 degree, (b) 90 degrees and (c) 180 degrees. The black solid line represents the results acquired by $2 \mathrm{D}$ circuit simulation. The black dash-dotted line represents the results acquired by transient electromagnetic simulation. The red solid line represents the result given by 1D circuit simulation. The consistency between the 2D circuit simulation results and transient electromagnetic simulation results proves the exactness of this circuit model.

technique is frequently employed on this part to enhance the explosive electron emission threshold [35].

Figure 8 displays the output transmission line voltage waveforms acquired by 1D, 2D circuit simulations, and transient electromagnetic simulation. Although they almost

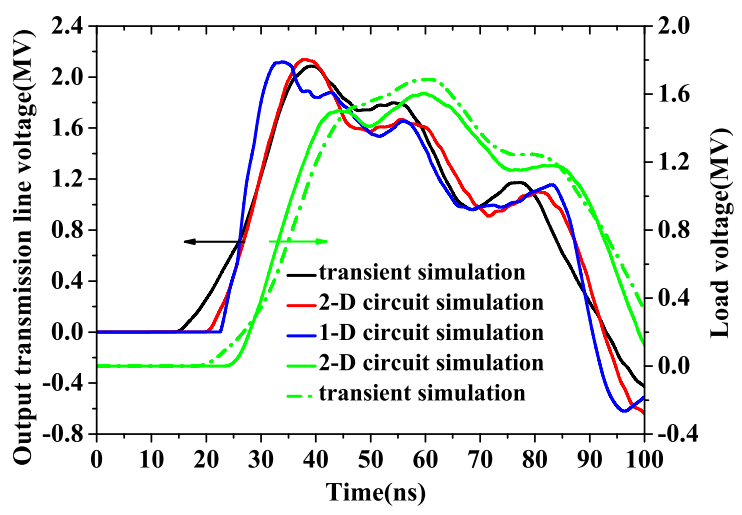

FIG. 8. Output transmission line and dummy load voltage waveforms acquired by $2 \mathrm{D}$ circuit simulation and transient electromagnetic simulation. The output transmission line voltage obtained by $1 \mathrm{D}$ circuit simulation (blue solid line) is also shown. The black solid line and red solid line represent the output transmission line voltage obtained by transient electromagnetic simulation and 2D circuit simulation, respectively, while the green solid line and green dash-dotted line represent the load voltage achieved by 2D circuit simulation and transient electromagnetic simulation, respectively. The output transmission line voltage probe is near the ground end. Comparing with 1D circuit simulation the output transmission line voltage waveform given by $2 \mathrm{D}$ circuit simulation agree with the result achieved by transient electromagnetic simulation better.

exhibit the same amplitudes, the rise time of voltage pulse acquired by transient simulation is $15.5 \mathrm{~ns}$ and is much greater than the result obtained by $1 \mathrm{D}$ circuit simulation which is about $6.9 \mathrm{~ns}$. The corresponding rise time achieved by $2 \mathrm{D}$ circuit simulation is $11.5 \mathrm{~ns}$. The load voltage waveforms are also presented in Fig. 8. The amplitude of load voltage is about 1.66 MV and is almost equal to the peak value of PFL output voltage, but the rise time of load voltage pulse is about $20.5 \mathrm{~ns}$ which is much greater than that of the PFL output voltage pulse.

The maximum voltage at the ground end of output transmission line is about 2.1 MV and is higher than the peak load voltage. This voltage decreasing is perhaps resulted from the fact that the output transmission line impedance does not match the load, and the transit time is $5.0 \mathrm{~ns}$ which is shorter than the rise time of voltage pulse. The electromagnetic wave will undergo multiple reflections in the output transmission line that serves as an inductor. The peak voltage will gradually decrease along the output transmission line because of the inductive drop.

\section{INFLUENCE OF IMPEDANCE MISMATCH ON THE POWER FLOW CONDITION IN THE INDUCTION CAVITY}

\section{A. Influence of the azimuthal transmission line impedance}

The ideal value of azimuthal transmission line impedance should roughly match the pulse forming section. 


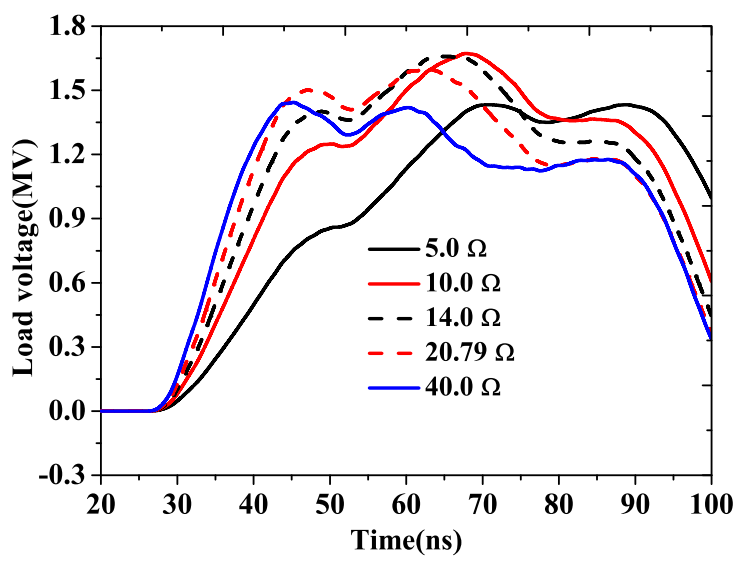

FIG. 9. Load voltage waveforms obtained by 2D circuit simulation when the azimuthal transmission line impedance is 5.0, 10.0, 14.0, 20.79 and $40.0 \Omega$.

However, not only the radial distance between the azimuthal transmission line high voltage electrode and inner induction cavity cylindrical shell is limited by oil insulation concern, but also the width of the azimuthal transmission line high voltage electrode is restricted since it will strongly influence the voltage distribution along the insulator rings [32]. Therefore, it is usually difficult to make the impedance of the azimuthal transmission line low enough and match the pulse forming section.

Figure 9 shows the load voltage waveforms with different azimuthal transmission line impedance. The amplitude of load voltage increases as the azimuthal transmission line impedance raises. It reaches the maximum as the azimuthal transmission line roughly matches the pulse forming section, and then decreases if the azimuthal transmission line impedance is sufficiently larger than that of the pulse forming section. However, the rise time of load voltage pulse decreases monotonically as the azimuthal transmission line impedance raises.

\section{B. Influence of the output transmission line impedance}

Figure 10 shows the load voltage waveforms with different output transmission line impedance. It can be seen that the rise time of load voltage pulse slightly increases as the output transmission line impedance increases, but they almost exhibit the same amplitudes. The peak value of load voltage is not sensitive to the actual output transmission line impedance. The voltage waveforms at the output transmission line section with different output transmission line impedance are shown in Fig. 11. It is clear that the rise time gradually decreases and the peak value increases a lot as the output transmission line impedance raises.

Commonly, the output transmission line is designed with stepped impedance profile along the power flow direction either in driver-matched or load-matched mode [28]. But this will not be followed if the electron emission is prohibited in the electrode gap. For instance, the output

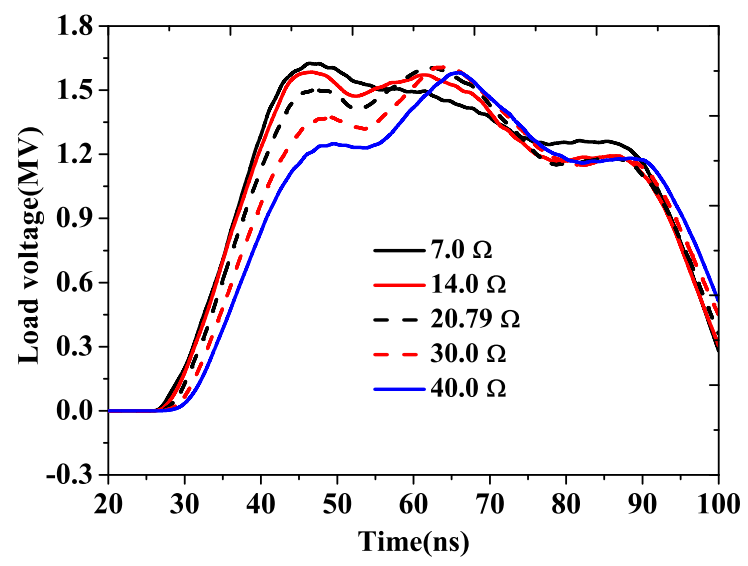

FIG. 10. Load voltage waveforms with different output transmission line impedance obtained by $2 \mathrm{D}$ circuit simulation.

transmission line of the Cygnus machine was conducted as a lossless vacuum transmission line and in positive polarity to drive a rod-pinch diode. The corresponding impedance of the output transmission line at each induction cavity was sufficiently larger than the summed impedance of upstream pulse forming sections in order to avoid electron emission from the negative outer electrode [36,37]. In this situation, not only the output transmission line impedance needs to be carefully chosen to make sure the cathode electric field at a safe level, but also the maximum voltages at the azimuthal transmission line, insulator stack, and radial feed gap sections will be higher than that of the pulse forming section. It should be taken into consideration when one designs an IVA machine like Cygnus.

\section{Influence of the load impedance}

The load voltage waveforms with different load impedance are shown in Fig. 12. It is obvious that the amplitude of load voltage gradually raises as the load impedance

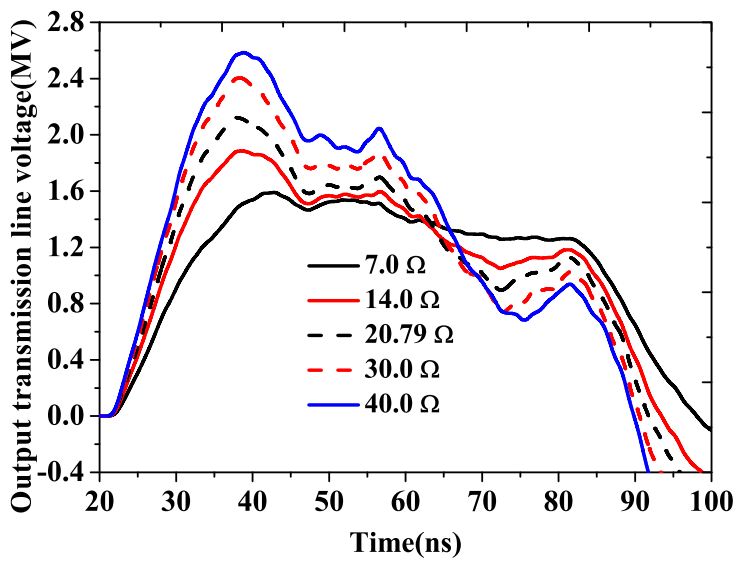

FIG. 11. Output transmission line voltage waveforms with different output transmission line impedance acquired by 2D circuit simulation. The voltage probe is near the ground end. 


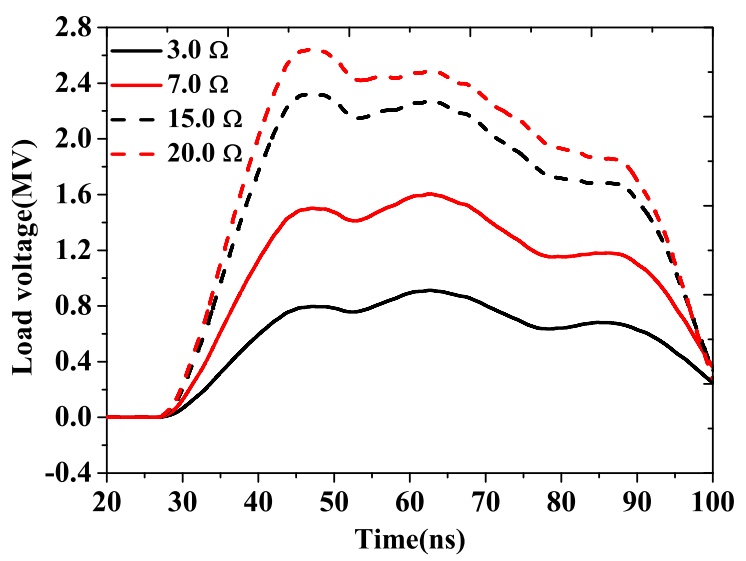

FIG. 12. Load voltage waveforms with different load impedance obtained by 2D circuit simulation.

increases. This is due to the fact that the IVA machine could be simply modeled by a Thevenin equivalent circuit [20]. The dummy load is driven by a voltage source whose internal resistor is equal to the water transmission line impedance and the source term is equal to twice the forward going voltage wave generated by the pulse forming section.
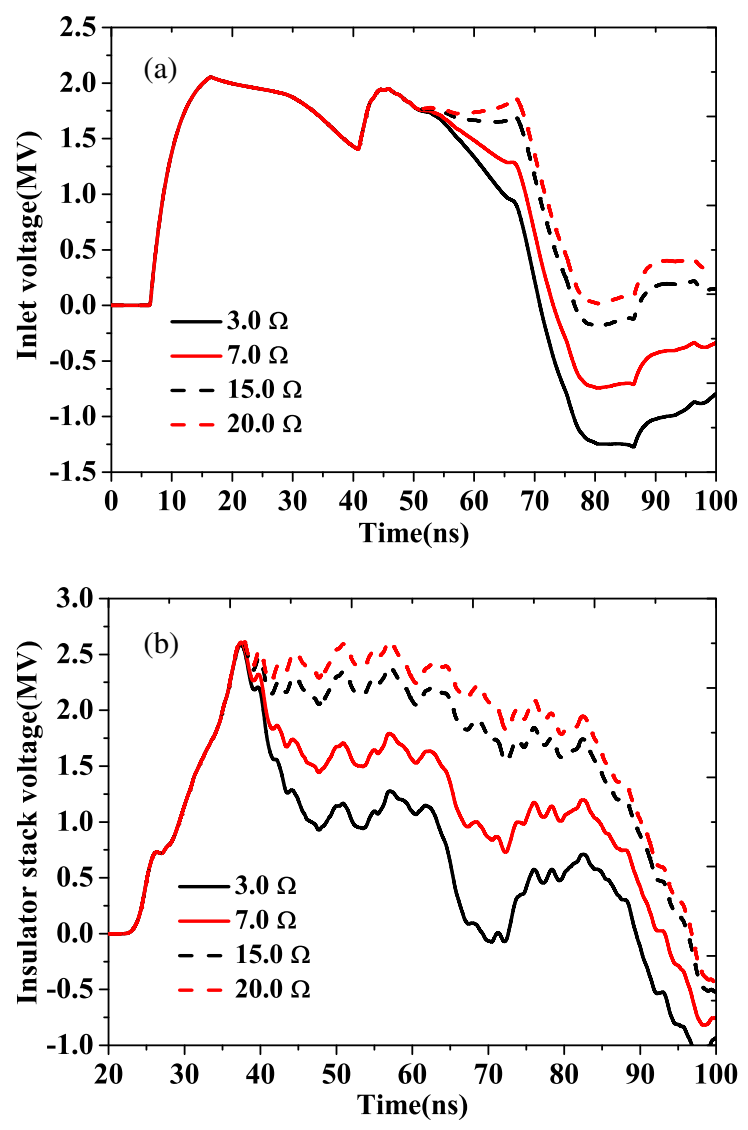

FIG. 13. (a) Oil elbow inlet and (b) insulator stack voltage waveforms with different load impedance acquired by $2 \mathrm{D}$ circuit simulation. The insulator stack voltage probe is at 0 degrees.
The voltage across the internal resistor gradually decreases and the voltage across the dummy load increases as the load impedance raises. However, most of the output transmission lines in IVA machines operate as MITL. The load impedance could not be greater than the output transmission line flow impedance even if the diode gap is sufficiently large [4]. In fact, many output transmission lines operate in load limited flow which means that the load impedance is less than the output transmission line flow impedance to reduce the loss current in the MITL region.

Figure 13 shows the oil elbow inlet and insulator stack voltage waveforms with different load impedance. Although the peak value is almost unchanged, the pulse duration increases as the load impedance raises. Therefore, it is desirable to estimate the electric field stress on the oil and insulator stack regions if we want to extend the IVA output voltage by redesigning the output transmission line impedance profile and increasing the load impedance.

\section{SUMMARY AND DISCUSSION}

A 2D circuit model of induction cavity has been proposed which could make circuit simulation more precise to investigate the power flow in the induction cell. The induction cavity is divided into many mesh cells in the cylindrical system. The power flow at the oil elbow and azimuthal transmission line sections is modeled with 1D transmission line elements. 2D transmission line elements are used to model the power flow inward the azimuthal transmission line. For the insulator stack and radial feed gap parts each mesh cell owns one radial element and one azimuthal element to model the radial and azimuthal directional power flow, respectively, while the axial and azimuthal elements are needed to represent the power flow in the output transmission line region. Transient electromagnetic simulation is employed to check out the exactness of the 2D circuit model. The voltage waveforms acquired by $2 \mathrm{D}$ circuit simulation and transient simulation at the oil elbow, azimuthal transmission line, insulator stack, output transmission line and dummy load could agree with each other very well. The power flow condition becomes worse if there exists impedance mismatch in the induction cavity, and this is numerically analyzed with this circuit model.

This circuit model is a useful tool for designing the IVA machine and induction cavity, as it could calculate the operating voltage at the azimuthal transmission line, insulator stack, radial feed gap and output transmission line regions accurately. However, the designing of an induction cavity could not be completed only by $2 \mathrm{D}$ circuit simulation. The electrostatic simulation is necessary to analyze the electric field in each key device. Meanwhile, because $2 \mathrm{D}$ circuit simulation is not able to calculate the magnetic field in the output transmission line region directly and accurately, the transient electromagnetic simulation is preferred to devise the configuration of azimuthal transmission line and connecting tab. Moreover, the high 
voltage electrode position of the azimuthal transmission line in the induction cavity will also influence the magnetic field distribution even though it keeps the same impedance profile. This could not be modeled by circuit simulation. Particle-in-cell (PIC) simulation is also required if the output transmission line operates as MITL, as it can calculate the loss current in the MITL region and electrical power transmission efficiency.

[1] J. Maenchen, G. Cooperstein, J. O’Malley, and I. Smith, Advances in pulsed power-driven radiography systems, Proc. IEEE 92, 1021 (2004).

[2] T. J. Goldsack, T. F. Bryant, P. F. Beech, S. G. Clough, G. M. Cooper, R. Davitt, R. D. Edwards, N. Kenna, J. McLean, A. G. Pearce, M. J. Phillips, K. P. Pullinger, D. J. Short, M. A. Sinclair, K. J. Thomas, J. R. Threadgold, M. C. Williamson, and K. Krushelnick, Multimegavolt multiaxis high-resolution flash $\mathrm{X}$-ray source development for a new hydrodynamics research facility at AWE Aldermaston, IEEE Trans. Plasma Sci. 30, 239 (2002).

[3] J. C. Zier, D. Mosher, R. J. Allen, R. J. Commisso, G. Cooperstein, D. D. Hinshelwood, S. L. Jackson, D. P. Murphy, P. F. Ottinger, A. S. Richardson, J. W. Schumer, S. B. Swanekamp, and B. V. Weber, High-power, photofission-inducing bremsstrahlung source for intense pulsed active detection of fissile material, Phys. Rev. ST Accel. Beams 17, 060401 (2014).

[4] D. D. Hinshelwood, P. F. Ottinger, J. W. Schumer, R. J. Allen, J. P. Apruzese, R. J. Commisso, G. Cooperstein, S. L. Jackson, D. P. Murphy, D. Phipps, S. B. Swanekamp, B. V. Weber, and F. C. Young, Ion diode performance on a positive polarity inductive voltage adder with layered magnetically insulated transmission line flow, Phys. Plasmas 18, 053106 (2011).

[5] J. A. Halbleib, T. W. L. Sanford, and J. W. Poukey, Radiation environment of Hermes III, IEEE Trans. Nucl. Sci. 35, 1282 (1988).

[6] J. J. Ramirez, K. R. Prestwich, E. L. Burgess, J. P. Furaus, R. A. Hamil, D. L. Johnson, T. W. L. Sanford, L. O. Seamons, L.X. Schneider, and G. A. Zawadzkas, The Hermes-III program, in Proceedings of the 6th IEEE International Pulsed Power Conference, edited by P. J. Turchi and B. H. Bernstein (IEEE, Piscataway, NJ, 1987), p. 294.

[7] J. P. Corley, J. A. Alexander, P. J. Pankuch, C. E. Heath, D. L. Johnson, J. J. Ramirez, and G. J. Denison, SABRE, A 10-MV linear induction accelerator, in Proceedings of the 8th IEEE International Pulsed Power Conference, edited by R. White and K. Prestwich (IEEE, Piscataway, NJ, 1991), p. 920.

[8] J. Fockler, K. Nielsen, H. Nishimoto, I. Smith, W. Bauer, K. Baumung, H. Bluhm, P. Hoppe, H. U. Karow, G. Kessler, W. Ratajczak, and D. Rusch, Design of a $6 \mathrm{MV}, 2$ TW positive ion driver using an induction adder, in Proceedings of the 9th IEEE International Pulsed Power
Conference, edited by K. Prestwich and W. Baker (IEEE, Piscataway, NJ, 1993), p. 431.

[9] I. D. Smith, V. L. Bailey, Jr., J. Fockler, J. S. Gustwiller, D. L. Johnson, J. E. Maenchen, and D. W. Droemer, Design of a radiographic integrated test stand (RITS) based on a voltage adder, to drive a diode immersed in a high magnetic field, IEEE Trans. Plasma Sci. 28, 1653 (2000).

[10] N. Bruner, T. Genoni, E. Madrid, D. Rose, D. Welch, K. Hahn, J. Leckbee, S. Portillo, B. Oliver, V. Bailey, and D. Johnson, Modeling particle emission and power flow in pulsed-power driven, nonuniform transmission lines, Phys. Rev. ST Accel. Beams 11, 040401 (2008).

[11] N. Bruner, T. Genoni, E. Madrid, D. Welch, K. Hahn, and B. Oliver, Excitation of voltage oscillations in an induction voltage adder, Phys. Rev. ST Accel. Beams 12, 070401 (2009).

[12] V. Carboni, P. Corcoran, J. Douglas, I. Smith, D. Johnson, R. White, B. Altes, R. Stevens, H. Nishimoto, R. Carlson, J. Smith, P. Ortega, J. Chavez, J. Maenchen, E. Ormond, D. Nelson, D. Henderson, T. Helvin, V. Mitton, and B. Anderson, Pulse power performance of the Cygnus 1, and 2 radiographic sources, in Proceedings of the 14th IEEE International Pulsed Power Conference, edited by M. Giesselmann and A. Neuber (IEEE, Piscataway, NJ, 2003), p. 905.

[13] K. Thomas et al., Status of the AWE Hydrus IVA fabrication, in Proceedings of the 18th IEEE International Pulsed Power Conference, edited by R. D. Curry and B. V. Oliver (IEEE, Piscataway, NJ, 2011), p. 1042.

[14] H. Wei, F. J. Sun, T. X. Liang, J. M. Guo, A. C. Qiu, P. T. Cong, J. H. Yin, Y. X. Hu, X. F. Jiang, Z. G. Wang, and T. F. Dang, Experimental study and electromagnetic model of a 1-MV induction voltage cavity, IEEE Trans. Plasma Sci. 43, 3359 (2015).

[15] J. J. Ramirez, K. R. Prestwich, D. L. Johnson, J. P. Corley, G. J. Denison, J. A. Alexander, T. L. Franklin, P. J. Pankuch, T. W. L. Sanford, T. J. Sheridan, L. L. Torrison, and G. A. Zawadzkas, Performance of the Hermes-III gamma ray simulator, in Proceedings of the 7th IEEE International Pulsed Power Conference, edited by B. H. Bernstein and J. P. Shannon (IEEE, Piscataway, NJ, 1989), p. 26.

[16] I. Smith, P. Corcoran, V. Carboni, V. Bailey, H. Kishi, D. L. Johnson, J. Maenchen, I. Molina, R. Carlson, D. Fulton, K. Hahn, J. Smith, D. Droemer, K. Thomas, M. Phillips, S. Croxon, R. Forgan, and I. D. Smith, Induction voltage adder architectures, and electrical characteristics, in Proceedings of the 14th IEEE International Pulsed Power Conference, edited by M. Giesselmann and A. Neuber (Ref. [12]), p. 371.

[17] J. J. Ramirez, K. R. Prestwich, and I. D. Smith, Highpower, short-pulse generators based on induction voltage adders, Proc. IEEE 80, 946 (1992).

[18] A. A. Kim, M. G. Mazarakis, V. A. Sinebryukhov, B. M. Kovalchuk, V. A. Visir, S. N. Volkov, F. Bayol, A. N. Bastrikov, V. G. Durakov, S. V. Frolov, V. M. Alexeenko, D. H. McDaniel, W. E. Fowler, K. LeChien, C. Olson, W. A. Stygar, K. W. Struve, J. Porter, and R. M. Gilgenbach, Development and tests of fast 1-MA linear transformer driver stages, Phys. Rev. ST Accel. Beams 12, 050402 (2009). 
[19] W. A. Stygar et al., Conceptual design of a $10^{13}-\mathrm{W}$ pulsedpower accelerator for megajoule-class dynamic-materialphysics experiments, Phys. Rev. Accel. Beams 19, 070401 (2016).

[20] R. J. Allen, R. J. Commisso, G. Cooperstein, P. F. Ottinger, and J. W. Schumer, Extension of the operating point of the Mercury IVA from 6 to $8 \mathrm{MV}$, in Proceedings of the 18th IEEE International Pulsed Power Conference, edited by R. D. Curry and B. V. Oliver (Ref. [13]), p. 1036.

[21] W. K. Zou, F. Guo, L. Chen, S. Y. Song, M. Wang, W. P. Xie, and J. J. Deng, Full circuit calculation for electromagnetic pulse transmission in a high current facility, Phys. Rev. ST Accel. Beams 17, 110401 (2014).

[22] L. Zhou, Z. H. Li, Z. Wang, C. Liang, M. J. Li, J. M. Qi, and Y. Y. Chu, Design of a 5-MA 100-ns lineartransformer-driver accelerator for wire array Z-pinch experiments, Phys. Rev. Accel. Beams 19, 030401 (2016).

[23] S. F. Glover, L. X. Schneider, K. W. Reed, G. E. Pena, J.-P. Davis, C. A. Hall, R. J. Hickman, K. C. Hodge, J. M. Lehr, D. J. Lucero, D. H. McDaniel, J. G. Puissant, J. M. Rudys, M. E. Sceiford, S. J. Tullar, D. M. Van De Valde, and F. E. White, Genesis: A 5-MA programmable pulsed-power driver for isentropic compression experiments, IEEE Trans. Plasma Sci. 38, 2620 (2010).

[24] P. A. Corcoran, B. A. Whitney, V. L. Bailey, I. D. Smith, W. A. Stygar, M. E. Savage, G. A. Rochau, J. E. Bailey, B. M. Jones, T. J. Nash, M. E. Sceiford, L. G. Schlitt, and J. W. Douglas, Circuit modeling techniques applied to ZR, in Proceedings of the 17th IEEE International Pulsed Power Conference, edited by F. Peterkin and R. Curry (IEEE, Piscataway, NJ, 2009), p. 150.

[25] P. A. Corcoran, Practical circuit models, and simulations using transmission lines, in Proceedings of the 18th IEEE International Pulsed Power Conference, edited by R. D. Curry and B. V. Oliver (Ref. [13]), p. 103.

[26] H. Wei, F. J. Sun, T. X. Liang, J. H. Yin, T. F. Dang, J. T. Zeng, P. T. Cong, and A.C. Qiu, Low voltage pulse injection test of a single-stage $1 \mathrm{MV}$ prototype induction voltage adder cell, Rev. Sci. Instrum. 85, 083506 (2014).

[27] B. V. Oliver, T. C. Genoni, D. L. Johnson, V. L. Bailey, P. Corcoran, I. Smith, J. E. Maenchen, I. Molina, and K. Hahn, Two and three-dimensional MITL power-flow studies on RITS, in Proceedings of the 14th IEEE International Pulsed Power Conference, edited by $\mathrm{M}$. Giesselmann and A. Neuber (Ref. [12]), p. 395.

[28] I. D. Smith, Induction voltage adders and the induction accelerator family, Phys. Rev. ST Accel. Beams 7, 064801 (2004).
[29] F. Guo, J. H. Jiang, B. Y. Gong, L. Chen, W. K. Zou, M. Wang, and W.P. Xie, Calculation of impedance of azimuthal transmission line in induction voltage adder accelerator, High Power Laser Part. Beams 28, 025001 (2016).

[30] C. A. Jennings, D. J. Ampleford, B. M. Jones, R. D. McBride, J. E. Bailey, M. C. Jones, M. R. Gomez, M. E. Cuneo, C. Nakhleh, W. A. Stygar, M. Savage, T. C. Wagoner, and J. K. Moore, Integration of MHD load models with circuit representations the $\mathrm{Z}$ generator, Sandia National Laboratories Report No. SAND2013-2690, 2013.

[31] D. L. Johnson, I. Smith, P. Corcoran, V. Bailey, J. Gustwiller, I. Molina, J. E. Maenchen, E. Hunt, E. Ormand, T. Helvin, S. Cordova, F. Wilkins, and R. Gignac, Tests of the first RITS PFL, and cell, in Proceedings of the 13th IEEE International Pulsed Power Conference, edited by B. Reinovsky and M. Newton (IEEE, Piscataway, NJ, 2001), p. 600.

[32] H. Wei, F. J. Sun, A. C. Qiu, J. T. Zeng, J. H. Yin, T. X. Liang, and Y.X. Hu, Optimized design of azimuthal transmission lines for the cell driven by two PFLs in induction voltage adders, IEEE Trans. Plasma Sci. 41, 2421 (2013).

[33] W. A. Stygar et al., Conceptual designs of two petawattclass pulsed-power accelerators for high-energy-densityphysics experiments, Phys. Rev. ST Accel. Beams 18, 110401 (2015).

[34] R. Burdt and R. D. Curry, Magnetic core test stand for energy loss and permeability measurements at a high constant magnetization rate and test results for nanocrystalline and ferrite materials, Rev. Sci. Instrum. 79, 094703 (2008).

[35] P. Flores, D. J. Henderson, D. E. Good, K. Hogge, C. V. Mitton, I. Molina, C. Naffziger, S. R. Codova, and E. C. Ormond, Anodizing of high electrically stressed components, in Proceedings of the 19th IEEE International Pulsed Power Conference, edited by B. V. Oliver, J. P. Verboncoeur, and M. T. Crawford (IEEE, Piscataway, NJ, 2013), p. 891.

[36] D. Weidenheimer, P. Corcoran, R. Altes, J. Douglas, H. Nishimoto, I. Smith, R. Stevens, D. L. Johnson, R. White, J. Gustwiller, J. E. Maenchen, P. Menge, R. Carlson, R. D. Fulton, G. Cooperstein, D. Droemer, and E. Hunt, Design of a driver for the Cygnus x-ray source, in Proceedings of the 13th IEEE International Pulsed Power Conference (Ref.), p. 591.

[37] E. C. Ormond, S. R. Cordova, I. Molina, D. S. Nelson, J. R. Smith, G. D. Corrow, M. D. Hansen, D. J. Henderson, and C. V. Mitton, Cygnus diverter switch analysis, IEEE Trans. Plasma Sci. 36, 2554 (2008). 